\title{
Interval changes in ROTEM values during cardiopulmonary bypass in pediatric cardiac surgery patients
}

\author{
Christopher F. Tirotta ${ }^{1 *}$ D, Richard G. Lagueruela', Daria Salyakina², Weize Wang ${ }^{2}$, Thomas Taylor ${ }^{2}$, Jorge Ojito ${ }^{3}$, \\ Kathleen Kubes ${ }^{3}$, Hyunsoo Lim³ ${ }^{3}$ Robert Hannan ${ }^{3}$ and Redmond Burke ${ }^{3}$
}

\begin{abstract}
Introduction: Rotational thromboelastometry (ROTEM) has been shown to reduce the need for transfused blood products in adult and pediatric cardiac surgery patients. However, similar evidence in newborns, neonates, and young infants is lacking. We quantified ROTEM value changes in pediatric patients on cardiopulmonary bypass (CPB) before, during and after blood product transfusion.

Methods: Each surgery had at least four interventions: initiating CPB; platelet administration during rewarming phase; post-CPB and following protamine and human fibrinogen concentrate (HFC) administration; and further component therapy if bleeding persisted and ROTEM indicated a deficiency. ROTEM assays were performed prior to surgery commencement, on CPB prior to platelet administration and following $38 \mathrm{~mL} / \mathrm{kg}$ platelets, and post-CPB after protamine and HFC administration. ROTEM assays were also performed in the post-CPB period after further blood component therapy administration.

Results: Data from 161 patients were analyzed. Regression models suggested significant changes in HEPTEM clotting time after all interventions. PLT administration during CPB improved HEPTEM a by $22.1^{\circ}(p<0.001)$ and FIBTEM maximum clot firmness (MCF) by $2.9 \mathrm{~mm}(\mathrm{p}<0.001)$. HFC administration after CPB termination significantly improved FIBTEM MCF by $2.6 \mathrm{~mm}(p<0.001)$. HEPTEM MCF significantly increased after $3 / 4$ interventions. HEPTEM a significantly decreased after two interventions and significantly increased after two interventions. Greatest perturbances in coagulation parameters occurred in patients $\leq 90$ days of age.

Conclusion: CPB induced profound perturbations in ROTEM values in pediatric cardiac surgery patients. ROTEM values improved following PLT and HFC administration. This study provides important clinical insights into ROTEM changes in pediatric patients after distinct interventions.
\end{abstract}

Keywords: ROTEM, Fibrinogen, RiaSTAP, Neonates, Infants, Cardiac surgery

\section{Introduction}

Rotational thromboelastometry (ROTEM, Tem International $\mathrm{GmbH}$, Munich, Germany) is an enhanced modification of thromboelastography (TEG, Haemonetics Corp., Braintree, MA, USA), first described in 1948 [1]. Both are point-of-care (POC) coagulation monitoring instruments that test the viscoelastic properties of whole blood [1]. TEG and ROTEM, while similar, have several

\footnotetext{
* Correspondence: christirotta@att.net

'Department of Anesthesia, The Heart Program, Nicklaus Children's Hospital

3100 S.W. 62nd Street, Miami, FL 33155, USA

Full list of author information is available at the end of the article
}

differences which can lead to discrepancies in results obtained, particularly for fibrin-based clotting measurements $[2,3]$.

A series of assays are employed with the ROTEM instrument. EXTEM provides information on the coagulation process via the extrinsic pathway and its interaction with thrombocytes in citrated blood; the reagent contains tissue factor and phospholipids used for extrinsic activation. HEPTEM provides information on the coagulation process via the intrinsic pathway in the presence of unfractionated heparin; this assay is similar to INTEM with the addition of heparinase to inactivate in

C The Author(s). 2019 Open Access This article is distributed under the terms of the Creative Commons Attribution 4.0 International License (http://creativecommons.org/licenses/by/4.0/), which permits unrestricted use, distribution, and 
vitro heparin. FIBTEM provides information on the fibrinogen level and quality of fibrin polymerization in citrated blood by inhibiting thrombocytes; the reagent contains thrombocyte inhibitor and recalcification reagent. APTEM provides information on clot firmness by inhibiting hyperfibrinolysis with aprotinin.

ROTEM has previously been validated for bedside use, with no significant differences in results compared with traditional laboratory assays, but a mean time saving of $11(8-16)$ minutes [4]. A prospective study by Ogawa et al compared values obtained using standard laboratory coagulation tests with ROTEM values in adult patients undergoing cardiac surgery, demonstrating that some ROTEM measurements could act as surrogates for standard coagulation tests [5]. However, although reference ROTEM values in pediatric patients have been described [6], similar data are lacking in newborns, neonates and young infants. The coagulation system functions very differently in these patient groups compared with older children and adults.

The aim of this study is to define and quantify how cardiopulmonary bypass (CPB) transfusion therapy with human fibrinogen concentrate (HFC), plateletpheresis (PLT), and other blood products affect ROTEM values.

\section{Methods}

\section{Study design}

A retrospective analysis of pediatric patients undergoing cardiac surgery requiring $\mathrm{CPB}$ at Nicklaus Children's Hospital, Miami, FL, USA between June 1, 2015 and August 31, 2017. This study received Institutional Review Board (IRB) exempt status from the Research Institute of Nicklaus Children's Hospital.

For each assay, the following parameters were considered: clotting time (s) (CT; time to reach $2 \mathrm{~mm}$ of amplitude from the test initiation); alpha ( $\alpha$; angle of the line between horizontal and the line from CT point to CFT point); clot formation time (s) (CFT; time between reaching $2 \mathrm{~mm}$ amplitude and $20 \mathrm{~mm}$ amplitude) and maximum clot firmness ( $\mathrm{mm}$ ) (MCF; maximum amplitude).

In accordance with standard institutional protocol, ROTEM assays were performed at the following times:

- ROTEM 1: baseline value obtained at the start of surgery prior to $\mathrm{CPB}$ initiation.

- ROTEM 2: during the rewarming phase of $\mathrm{CPB}$, prior to PLT administration (occurring after the administration of blood products in the priming phase).

- ROTEM 3: on CPB after PLT administration.

- ROTEM 4: after CPB termination, and after protamine and HFC administration.
- ROTEM 5: after the administration of other blood products if bleeding persisted and ROTEM 4 indicated a deficiency.

Each surgery had at least four interventions.

- Intervention I: when initiating $\mathrm{CPB}$, for patients less than $10 \mathrm{~kg}$, the pump was primed with $1 \mathrm{U}$ PRBC. If the patient was less than $3 \mathrm{~kg}, 50 \mathrm{~mL}$ of PLT were added to the prime. If the patient was antithrombin III deficient or the heparin management system revealed heparin resistance (based on pre-operative values), $60 \mathrm{~mL}$ of fresh frozen plasma (FFP) was added to the prime.

- Intervention II: administration of PLT given during the rewarming phase of $\mathrm{CPB}$ with a median dose of $38 \mathrm{~mL} / \mathrm{kg}$.

- Intervention III: administration of Protamine, and HFC (70 mg/kg; RiaSTAP, CSL Behring, Marburg, Germany) after CPB termination.

- Intervention IV: further component therapy if bleeding persisted after the HFC and ROTEM indicated a deficiency. This could be further HFC, PLT, FFP or cryoprecipitate

\section{Statistical analysis methods}

Descriptive statistics, including sample median and interquartile range, were calculated for patients' characteristics at baseline and at the four intervention time points, for blood or coagulation products (packed red blood cells [PRBC], platelets, FFP, Cell Saver [CS], Cryoprecipitate, phlebotomized blood [PB], or HFC). Calculations were performed for both the overall population and subgroups stratified by age ( $\leq 90$ days; $>90$ days and $\leq$ 2 years of age; and $>2$ years of age). At intervention I, PRBC and FFP could be provided twice, so the cumulative dose for each was used when calculating medians and interquartile ranges. Kruskal-Wallis/Mann Whitney U tests were performed to identify any significant difference in the distribution of each variable by age groups.

Regression analysis was performed to adjust for the variation in per patient blood product administration. Generalized estimation equation (GEE) modeling was applied to predict HEPTEM CT, HEPTEM MCF and FIBTEM MCF (when data were available) to quantify the change in ROTEM values after each wave of platelets/HFC administration. Generalized linear mixed modeling with a beta distribution was employed to model the proportion of HEPTEM $\alpha$ in the $0-90^{\circ}$ range. Proportions estimated by the model were then rescaled to the original scale of measurement $\left(0-90^{\circ}\right)$ for ease of interpretation of regression results.

Predictors included in the GEE and beta regression models were age group, before/after platelet or HFC 
administration, administration of each of the blood products, and the interaction between age group and the before/after platelet or HFC administration predictors.

Results of HEPTEM CT $>1800 \mathrm{~s}$ were excluded from regression analysis due to outliers affecting the assumptions and convergence of the GEE models. Predicted marginal means (SAS PROC LSMEANS) with 95\% confidence intervals (CIs) of ROTEM values (HEPTEM CT, HEPTEM MCF, FIBTEM MCF, and HEPTEM $\alpha$ ) were calculated by age group for timepoints before and after each wave of administration, and the change between timepoints. Bonferroni-corrected $p$-values were calculated for the changes in ROTEM values by age group.

All data analysis was performed at a 0.05 level of significance and conducted using SAS Enterprise Guide 7.1 (SAS Institute, Cary, NC, USA).

\section{Results}

A total of 161 pediatric patients (median age, 214 days; interquartile range, 1324 days) were included in the study. Of these patients, $26 \%$ were $\leq 90$ days of age, $40 \%$ were between 90 days and 2 years of age, and 34\% were older than 2 years. Table 1 presents descriptive statistics and comparison between the three age groups. Some parameters varied significantly between these age groups during the surgery. The median bypass time was the longest for youngest patients $(146.5 \mathrm{~min})$, and shortest in the $>90$ days $\leq 2$ years group $(87.0 \mathrm{~min} ; p<0.001)$. The youngest patients also had the lowest median low temperature during the surgery, relative to the two older groups $(p<0.001)$. Because of the very small sample size, there were no statistically significant differences between the three groups in cross clamp time, incidence of regional low flow perfusion or deep hypothermic circulatory arrest;

Table 1 Descriptive statistics of patients' characteristics and interventions $(N=161)$

\begin{tabular}{|c|c|c|c|c|c|c|c|c|c|c|c|c|c|}
\hline & \multirow{2}{*}{\multicolumn{3}{|c|}{ Overall }} & \multicolumn{9}{|c|}{ Age Groups } & \multirow[t]{3}{*}{$p^{b}$} \\
\hline & & & & \multicolumn{3}{|c|}{90 days or less } & \multicolumn{3}{|c|}{ Older than 90 days to 2 years } & \multicolumn{3}{|c|}{ Older than 2 years } & \\
\hline & $\overline{N^{a}}$ & Median & IQR & $\mathrm{N}$ & Median & $\overline{\mathrm{IQR}}$ & $\mathrm{N}$ & Median & IQR & $\bar{N}$ & Median & IQR & \\
\hline Weight (kg) & 161 & 6.4 & 12.0 & 42 & 3.2 & 0.8 & 64 & 6.2 & 2.8 & 55 & 24.0 & 38.0 & $<0.001$ \\
\hline Height (cm) & 161 & 65.0 & 47.0 & 42 & 50.3 & 5.0 & 64 & 64.0 & 10.3 & 55 & 127.0 & 57.0 & $<0.001$ \\
\hline Age (days) & 161 & 214.0 & 1324.0 & 42 & 9.5 & 38.0 & 64 & 193.5 & 147.5 & 55 & 2649.0 & 3582.0 & $<0.001$ \\
\hline Bypass (min) & 161 & 106.0 & 74.0 & 42 & 146.5 & 64.0 & 64 & 87.0 & 46.0 & 55 & 106.0 & 73.0 & $<0.001$ \\
\hline Cross clamp time (min) & 140 & 62.0 & 49.5 & 42 & 82.5 & 56.0 & 52 & 55.5 & 38.0 & 46 & 58.5 & 46.0 & 0.053 \\
\hline Regional low flow perfusion (min) & 24 & 102.0 & 66.5 & 22 & 114.5 & 57.0 & 2 & 31.5 & 55.0 & 0 & 0.0 & 0.0 & 0.089 \\
\hline Deep hypothermic circulatory arrest (min) & 15 & 15.0 & 41.0 & 12 & 9.5 & 38.0 & 3 & 27.0 & 43.0 & 0 & 0.0 & 0.0 & 0.829 \\
\hline Low temperature $(c)$ & 159 & 29.1 & 7.2 & 42 & 17.8 & 7.8 & 64 & 29.4 & 3.3 & 53 & 30.1 & 4.6 & $<0.001$ \\
\hline \multicolumn{14}{|l|}{ Intervention I } \\
\hline Packed red blood cells $(\mathrm{mL} / \mathrm{kg})$ & 108 & 62.0 & 52.0 & 42 & 96.5 & 77.0 & 62 & 44.5 & 23.0 & 4 & 13.5 & 14.5 & $<0.001$ \\
\hline Plateletpheresis (mL/kg) & 13 & 23.0 & 8.0 & 10 & 22.0 & 8.0 & 3 & 26.0 & 12.0 & 0 & 0.0 & 0.0 & 0.934 \\
\hline Fresh frozen plasma (mL/kg) & 27 & 24.0 & 9.0 & 21 & 23.0 & 7.0 & 5 & 26.0 & 8.0 & 1 & 6.0 & 0.0 & 0.055 \\
\hline \multicolumn{14}{|l|}{ Intervention || } \\
\hline Plateletpheresis (mL/kg) & 95 & 38.0 & 29.0 & 40 & 56.0 & 17.5 & 54 & 29.5 & 12.0 & 1 & 18.0 & 0.0 & $<0.001$ \\
\hline \multicolumn{14}{|l|}{ Intervention III } \\
\hline $\mathrm{HFC}(\mathrm{mg} / \mathrm{kg})$ & 129 & 70.0 & 2.0 & 40 & 70.0 & 1.0 & 58 & 70.0 & 2.0 & 31 & 68.0 & 7.0 & $<0.001$ \\
\hline \multicolumn{14}{|l|}{ Intervention IV } \\
\hline HFC (mg/kg) & 45 & 70.0 & 1.0 & 24 & 70.0 & 1.0 & 19 & 70.0 & 1.0 & 2 & 46.5 & 47.0 & 0.229 \\
\hline Plateletpheresis (mL/kg) & 81 & 13.0 & 11.0 & 40 & 19.0 & 13.5 & 40 & 9.0 & 3.5 & 1 & 5.0 & 0.0 & $<0.001$ \\
\hline Cell saver $(\mathrm{mL} / \mathrm{kg})$ & 151 & 17.0 & 19.0 & 36 & 33.0 & 22.0 & 62 & 19.0 & 9.0 & 53 & 5.0 & 4.0 & $<0.001$ \\
\hline Fresh frozen plasma $(\mathrm{mL} / \mathrm{kg})$ & 22 & 19.5 & 13.0 & 17 & 20.0 & 9.0 & 4 & 15.5 & 51.5 & 1 & 3.0 & 0.0 & 0.226 \\
\hline Packed red blood cells (mL/kg) & 18 & 20.5 & 22.0 & 12 & 25.0 & 13.0 & 4 & 6.5 & 10.0 & 2 & 8.0 & 6.0 & 0.008 \\
\hline Cryoprecipitate (mL/kg) & 2 & 10.0 & 0.0 & 1 & 10.0 & 0.0 & 1 & 10.0 & 0.0 & 0 & 0.0 & 0.0 & NA \\
\hline Phlebotomized blood (mL/kg) & 19 & 10.0 & 4.0 & 0 & 0.0 & 0.0 & 0 & 0.0 & 0.0 & 19 & 10.0 & 4.0 & NA \\
\hline
\end{tabular}

${ }^{\mathrm{a}} \mathrm{N}$ is number of patients received the procedure/blood product. Descriptive statistics excluded patients who did not receive the procedure/product ${ }^{b} p$ values from Kruskal Wallis test by age groups. When there was no patient in the age group, Mann Whitney $U$ test was applied. If $p=N A$ (Not Available), sample size was too small to perform the test $H F C$ human fibrinogen concentrate, IQR interquartile range $p$ values in bold represent statistical significance 
however, there was a tendency towards higher median values in younger children.

Results from GEE and beta regression models suggested significant overall changes in several intervention waves for all four ROTEM measurements (Fig. 1, Table 2). Specifically, significant changes were observed in HEPTEM CT and HEPTEM $\alpha$ after all four interventions; and HEPTEM MCF and FIBTEM MCF after three interventions. Moreover, mean fibrinogen levels decreased 162.1 $\mathrm{mg} / \mathrm{dL}$ (range $136.5-187.7 \mathrm{mg} / \mathrm{dL}, p<0.001$ ) after Intervention $\mathrm{I}$, increased $69.8 \mathrm{mg} / \mathrm{dL}$ (range $58.3-81.2 \mathrm{mg} / \mathrm{dL}$, $p<0.001$ ) after Intervention II, and increased $73.1 \mathrm{mg} / \mathrm{dL}$ (range 43.1-103.1, $p=.001$ ) after Intervention III.

\section{Analysis of ROTEM parameters by age group Intervention I: CBP priming and initiation}

A significant increase in HEPTEM CT was observed for patients aged $\leq 90$ days (estimated mean change [EMC]: 99.9, 95\% CI: $64.0-135.8, p<0.001)$ and those $>90$ days and $\leq 2$ years (EMC: 83.5 , 95\% CI: 66.8-100.3, $p<0.001$ ). There was no significant change in mean HEPTEM CT among patients $>2$ years $(p=0.175$, Table 3; Fig. 2$)$.
HEPTEM MCF was significantly decreased in all three groups $(p<0.001)$. Compared with patients in the two older age groups, patients $\leq 90$ days had a greater decrease in mean HEPTEM MCF (EMC: -32.1, 95\% CI: 35.3--28.8). Similarly, FIBTEM MCF and HEPTEM $\alpha$ were significantly reduced in the three age groups, with the youngest group having the greatest decrease (FIBTEM MCF EMC: -13.3, 95\% CI: - 15.9--10.7, $p<0.001$; HEPTEM $\alpha$ EMC: $-40.4,95 \%$ CI: $-50.4--30.5, p<0.001)$.

\section{Intervention II: PLT administration during the rewarming phase of $C P B$}

Patients in the two younger age groups had a significant decrease in HEPTEM CT (EMCs: -84.0 and - 72.7, 95\% CIs: $-161.1--7.0$ and -90.8 to -54.6 , respectively, both $p<0.001$ ), while no significant change was found in patients $>2$ years $(p=1.000)$. HEPTEM MCF rose significantly in the two younger age groups (EMCs: 25.2 and 17.3, 95\% CIs: 21.5-29.0 and 13.8-20.9, respectively, both $p<0.001$ ), whereas no significant change was seen in patients $>2$ years $(p=1.000)$. Consistent results were seen in changes of FIBTEM MCF and HEPTEM $\alpha$, with

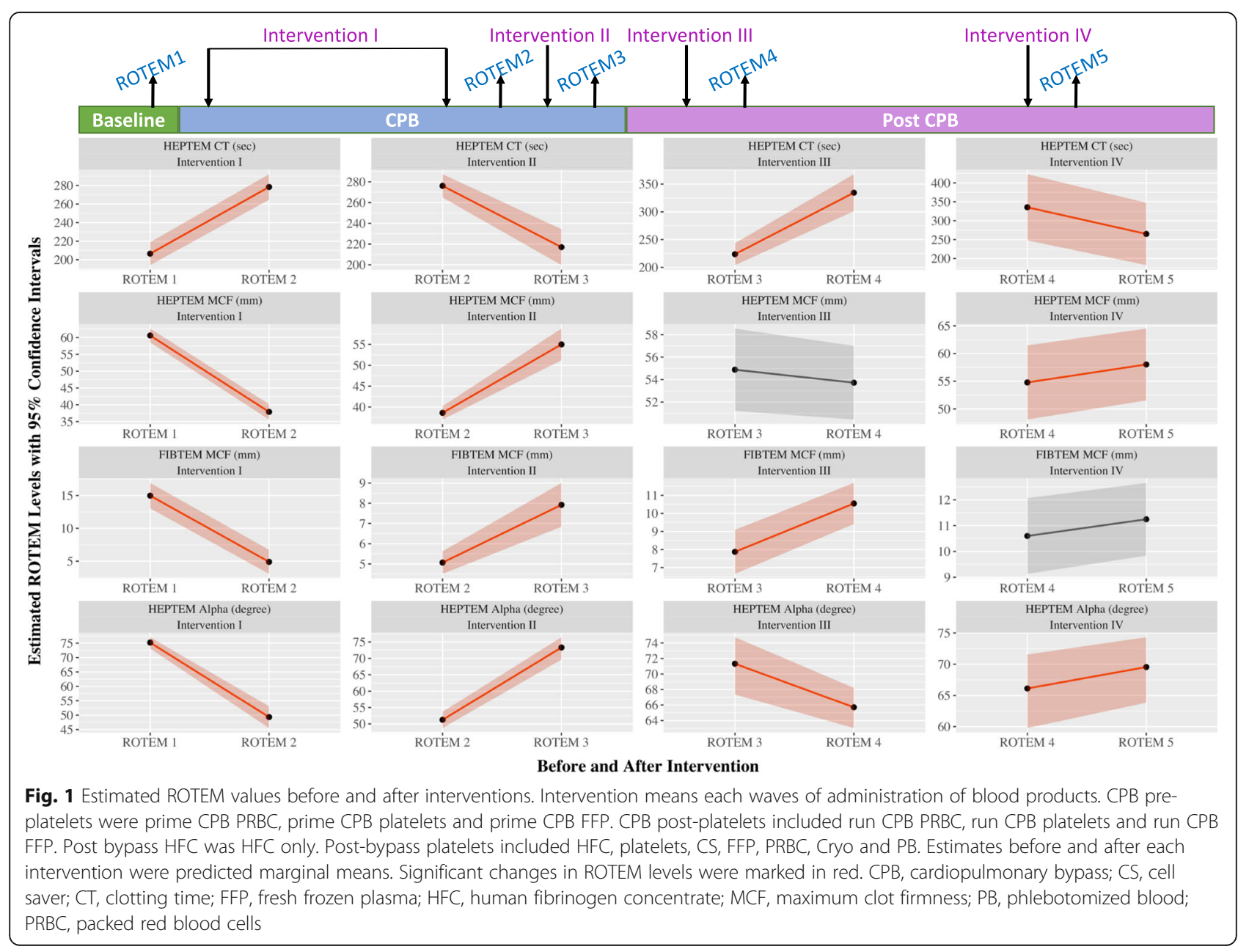


Table 2 Estimated changes in ROTEM values and fibrinogen levels after four waves of blood product transfusion (intervention)

\begin{tabular}{|c|c|c|c|c|c|c|}
\hline \multirow[t]{2}{*}{ Outcome } & \multirow[t]{2}{*}{ Intervention } & \multicolumn{4}{|c|}{ Estimates } & \multirow[t]{2}{*}{$p^{b}$} \\
\hline & & $\overline{N^{a}}$ & Before $(95 \% \mathrm{Cl})$ & After $(95 \% \mathrm{Cl})$ & Change $(95 \% \mathrm{Cl})$ & \\
\hline \multirow[t]{4}{*}{ HEPTEM CT (sec) } & Intervention I & 266 & $206.5(194.2,218.9)$ & $278.4(264.6,292.2)$ & $71.9(59.8,83.9)$ & $<0.001$ \\
\hline & Intervention || & 206 & $276.2(264.9,287.4)$ & $217.0(199.7,234.4)$ & $-59.1(-79.3,-39.0)$ & $<0.001$ \\
\hline & Intervention ||| & 219 & $223.7(204.0,243.4)$ & $334.4(301.1,367.8)$ & $110.8(77.7,143.8)$ & $<0.001$ \\
\hline & Intervention IV & 189 & $335.6(248.1,423.1)$ & $265.1(182.5,347.7)$ & $-70.5(-102.8,-38.2)$ & $<0.001$ \\
\hline \multirow[t]{4}{*}{ HEPTEM MCF $(\mathrm{mm})$} & Intervention I & 266 & $60.6(58.5,62.7)$ & $37.9(35.6,40.2)$ & $-22.7(-24.2,-21.2)$ & $<0.001$ \\
\hline & Intervention || & 205 & $38.6(37.0,40.2)$ & $55.0(51.2,58.8)$ & $16.4(12.4,20.4)$ & $<0.001$ \\
\hline & Intervention ||| & 217 & $54.9(51.2,58.5)$ & $53.7(50.4,57.0)$ & $-1.2(-3.7,1.4)$ & 0.380 \\
\hline & Intervention IV & 188 & $54.8(48.1,61.5)$ & $58.0(51.5,64.5)$ & $3.3(1.1,5.4)$ & 0.003 \\
\hline \multirow[t]{4}{*}{ FIBTEM MCF (mm) } & Intervention I & 255 & $15.0(13.0,16.9)$ & $4.9(3.1,6.7)$ & $-10.1(-11.0,-9.2)$ & $<0.001$ \\
\hline & Intervention || & 196 & $5.1(4.5,5.6)$ & $7.9(6.8,9.0)$ & $2.9(1.8,4.0)$ & $<0.001$ \\
\hline & Intervention III & 219 & $7.9(6.7,9.1)$ & $10.5(9.4,11.7)$ & $2.7(2.0,3.4)$ & $<0.001$ \\
\hline & Intervention IV & 190 & $10.6(9.1,12.1)$ & $11.2(9.8,12.7)$ & $0.6(-0.3,1.6)$ & 0.197 \\
\hline \multirow[t]{4}{*}{ HEPTEM a $\left({ }^{\circ}\right)$} & Intervention I & 265 & $75.2(73.1,77.1)$ & $49.3(45.5,53.1)$ & $-25.9(-30.8,-21.0)$ & $<0.001$ \\
\hline & Intervention || & 204 & $51.2(48.7,53.7)$ & $73.3(69.6,76.5)$ & $22.1(16.8,27.4)$ & $<0.001$ \\
\hline & Intervention III & 217 & $71.3(67.4,74.7)$ & $65.7(63.0,68.2)$ & $-5.6(-8.5,-2.7)$ & $<0.001$ \\
\hline & Intervention IV & 188 & $66.1(59.8,71.6)$ & $69.6(63.8,74.3)$ & $3.4(1.0,5.9)$ & 0.005 \\
\hline
\end{tabular}

${ }^{a} \mathrm{~N}$ was number of observations used for each model. All the models were controlled for age groups, whether each of the interventions was received, as well as interaction between age groups and before or after the intervention

${ }^{\mathrm{b}}$ Generalized linear mixed model using beta distribution was applied to predict a $\left({ }^{\circ}\right)$, while generalized estimation equation was used for other outcomes. Estimates were predicted marginal means

Cl confidence interval, CT clotting time, MCF maximum clot firmness

$p$ values in bold represent statistical significance

the two younger groups demonstrating significant increases $(p<0.001)$, whereas the change in patients $>2$ years was not significant $(p>1.000)$.

\section{Intervention III: protamine and HFC administration after the termination of $C P B$}

HEPTEM CT increased dramatically in patients $\leq 90$ days old (EMC: 234.9, 95\% CI: 89.9-379.8, $p<0.001$ ), while it also increased significantly but less notably in patients > 90 days and $\leq 2$ years (EMC: 81.0, 95\% CI: $51.1-110.9, p<0.001)$. No significant change in HEPTEM CT was found in patients $>2$ years old $(p=0.401)$. HEPTEM MCF did not significantly differ after Intervention III in all three age groups $(p=1.000)$. In contrast, FIBTEM MCF was significantly higher, with an estimated increase of $2.4 \mathrm{~mm}$ (95\% CI: 0.9-3.9, $p<$ $0.001)$ in patients $\leq 90$ days old, $2.2 \mathrm{~mm}$ (95\% CI: $1.0-3.4$, $p<0.001)$ in patients $>90$ days and $\leq 2$ years, and $3.4 \mathrm{~mm}$ (95\% CI: $0.9-5.9, p<0.001$ ) in patients $>2$ years old. HEPTEM $\alpha$ decreased significantly in the two younger age groups $(p<0.001)$, while no significant change in HEPTEM $\alpha$ was found in patients $>2$ years $(p=1.000)$.

Intervention IV: further component therapy if bleeding persists HEPTEM CT significantly dropped by $152.3 \mathrm{~s}$ (EMC: $-152.3,95 \%$ CI: $-265.6--39.0, p=0.001)$ for patients $\leq 90$ days, whereas it decreased by $43.8 \mathrm{~s}$ (EMC: -43.8 , 95\% CI: $-76.1--11.5, \mathrm{p}=0.001)$ for patients $>2$ years. The change in HEPTEM CT for patients $\geq 90$ days to $\leq 2$ years was not significantly different from $0(\mathrm{p}=1.000)$. No significant change was found in HEPTEM MCF or FIBTEM MCF in all three age groups $(p>0.05)$. HEPTEM $\alpha$ increased by $5.7^{\circ}$ (EMC: $5.7,95 \%$ CI: $0.3-11.2$, $p=0.038)$ in the youngest patients. There was no significant change in HEPTEM $\alpha$ in patients $>90$ days $(p=1.000)$.

\section{Discussion}

The results of this study demonstrate that there are predictable and quantifiable changes in ROTEM values following administration of PLT and HFC during CPB surgery in newborns, neonates and young infants. $\mathrm{CPB}$ negatively and significantly impacts all ROTEM values assessed in this analysis (HEPTEM $\alpha$, HEPTEM CT, HEPTEM MCF, and FIBTEM MCF). The greatest perturbances in coagulation parameters occurred consistently in patients $\leq 90$ days of age, followed by patients $>90$ days to $\leq 2$ years of age; while patients older than 2 years were affected the least.

Platelet transfusion (Intervention II) significantly improved all ROTEM parameters. Prolongation of the median HEPTEM CT at timepoints during CBP 
Table 3 Estimated average changes in ROTEM values after four waves of interventions (blood product transfusions)

\begin{tabular}{|c|c|c|c|c|c|c|c|}
\hline \multirow[t]{2}{*}{ Outcome } & \multirow[t]{2}{*}{ Intervention } & \multirow[t]{2}{*}{$\mathrm{N}^{\mathrm{a}}$} & \multirow[t]{2}{*}{ Age Group } & \multicolumn{3}{|c|}{ Estimated ROTEM Values } & \multirow[t]{2}{*}{$p^{b, c}$} \\
\hline & & & & Before $(95 \% \mathrm{Cl})$ & After $(95 \% \mathrm{Cl})$ & Change $(95 \% \mathrm{Cl})$ & \\
\hline \multirow[t]{12}{*}{ HEPTEM CT (sec) } & Intervention I & 266 & \multirow[t]{4}{*}{90 days or less } & $223.2(202.0,244.5)$ & $323.1(295.9,350.4)$ & $99.9(64.0,135.8)$ & $<0.001$ \\
\hline & Intervention || & 206 & & $305.9(282.3,329.4)$ & $221.8(175.3,268.3)$ & $-84.0(-161.1,-7.0)$ & 0.021 \\
\hline & Intervention ||| & 219 & & $231.5(185.4,277.5)$ & $466.3(382.7,550.0)$ & $234.9(89.9,379.8)$ & $<0.001$ \\
\hline & Intervention IV & 189 & & $444.2(345.7,542.7)$ & $291.9(221.9,361.9)$ & $-152.3(-265.6,-39.0)$ & 0.001 \\
\hline & Intervention I & 266 & \multirow[t]{4}{*}{ Older than 90 days to 2 years } & $210.2(187.5,232.9)$ & $293.7(272.1,315.3)$ & $83.5(66.8,100.3)$ & $<0.001$ \\
\hline & Intervention || & 206 & & $280.0(266.9,293.2)$ & $207.3(194.9,219.7)$ & $-72.7(-90.8,-54.6)$ & $<0.001$ \\
\hline & Intervention ||| & 219 & & $217.1(199.7,234.5)$ & $298.1(269.2,327.0)$ & $81.0(51.1,110.9)$ & $<0.001$ \\
\hline & Intervention IV & 189 & & $294.8(201.3,388.2)$ & $279.4(176.5,382.2)$ & $-15.4(-100.6,69.8)$ & 1.000 \\
\hline & Intervention I & 266 & \multirow[t]{4}{*}{ Older than 2 years } & $186.1(166.2,206.1)$ & $218.3(191.9,244.7)$ & $32.2(-5.3,69.6)$ & 0.175 \\
\hline & |ntervention || & 206 & & $242.7(219.9,265.4)$ & $207.3(194.9,219.7)$ & $-20.7(-66.7,25.4)$ & 1.000 \\
\hline & Intervention III & 219 & & $222.5(204.7,240.4)$ & $238.9(217.5,260.3)$ & $16.4(-5.3,38.1)$ & 0.401 \\
\hline & Intervention IV & 189 & & $267.8(159.1,376.4)$ & $223.9(122.0,325.9)$ & $-43.8(-76.1,-11.5)$ & 0.001 \\
\hline \multirow[t]{12}{*}{ HEPTEM MCF (mm) } & Intervention I & 266 & \multirow[t]{4}{*}{90 days or less } & $62.2(58.6,65.7)$ & $30.1(26.5,33.7)$ & $-32.1(-35.3,-28.8)$ & $<0.001$ \\
\hline & Intervention || & 205 & & $32.8(30.1,35.5)$ & $58.0(55.3,60.7)$ & $25.2(21.5,29.0)$ & $<0.001$ \\
\hline & Intervention III & 217 & & $57.0(53.5,60.6)$ & $55.2(51.0,59.3)$ & $-1.9(-6.8,3.1)$ & 1.000 \\
\hline & Intervention IV & 188 & & $54.7(47.6,61.8)$ & $58.7(52.0,65.5)$ & $4.0(-1.8,9.9)$ & 0.659 \\
\hline & Intervention I & 266 & \multirow[t]{4}{*}{ Older than 90 days to 2 years } & $59.9(56.4,63.5)$ & $36.6(33.1,40.2)$ & $-23.3(-25.5,-21.1)$ & $<0.001$ \\
\hline & Intervention || & 205 & & $38.7(36.6,40.8)$ & $56.0(53.7,58.4)$ & $17.3(13.8,20.9)$ & $<0.001$ \\
\hline & Intervention ||I & 217 & & $55.0(51.8,58.3)$ & $55.6(52.2,59.0)$ & $0.6(-2.5,3.7)$ & 1.000 \\
\hline & Intervention IV & 188 & & $56.4(49.6,63.2)$ & $58.3(50.9,65.7)$ & $1.9(-3.8,7.6)$ & 1.000 \\
\hline & Intervention I & 266 & \multirow[t]{4}{*}{ Older than 2 years } & $59.7(56.3,63.1)$ & $47.0(42.4,51.6)$ & $-12.7(-18.3,-7.2)$ & $<0.001$ \\
\hline & Intervention II & 205 & & $44.3(40.6,48.0)$ & $51.0(40.6,61.4)$ & $6.7(-10.4,23.8)$ & 1.000 \\
\hline & Intervention ||| & 217 & & $52.6(45.2,60.0)$ & $50.4(46.6,54.2)$ & $-2.2(-12.1,7.8)$ & 1.000 \\
\hline & Intervention IV & 188 & & $53.2(45.5,61.0)$ & $57.1(49.3,64.8)$ & $3.9(-0.9,8.6)$ & 0.250 \\
\hline \multirow[t]{12}{*}{ FIBTEM MCF (mm) } & Intervention I & 255 & \multirow[t]{4}{*}{90 days or less } & $17.0(13.2,20.8)$ & $3.7(0.3,7.0)$ & $-13.3(-15.9,-10.7)$ & $<0.001$ \\
\hline & Intervention || & 196 & & $4.3(3.4,5.3)$ & $8.5(7.2,9.8)$ & $4.2(2.6,5.7)$ & $<0.001$ \\
\hline & Intervention III & 219 & & $8.2(6.7,9.7)$ & $10.6(9.1,12.1)$ & $2.4(0.9,3.9)$ & $<0.001$ \\
\hline & Intervention IV & 190 & & $10.4(8.8,12.0)$ & $12.5(10.8,14.2)$ & $2.1(-0.7,4.9)$ & 0.396 \\
\hline & Intervention I & 255 & \multirow[t]{4}{*}{ Older than 90 days to 2 years } & $15.1(11.0,19.2)$ & $4.3(0.8,7.7)$ & $-10.9(-13.1,-8.6)$ & $<0.001$ \\
\hline & Intervention || & 196 & & $4.6(3.8,5.5)$ & $8.3(7.4,9.1)$ & $3.6(2.8,4.4)$ & $<0.001$ \\
\hline & Intervention III & 219 & & $7.9(6.8,9.0)$ & $10.1(8.9,11.3)$ & $2.2(1.0,3.4)$ & $<0.001$ \\
\hline & Intervention IV & 190 & & $10.2(8.5,11.9)$ & $11.7(9.6,13.8)$ & $1.5(-0.7,3.7)$ & 0.736 \\
\hline & Intervention I & 255 & \multirow[t]{4}{*}{ Older than 2 years } & $12.8(9.2,16.5)$ & $6.8(3.8,9.7)$ & $-6.1(-8.5,-3.7)$ & $<0.001$ \\
\hline & Intervention || & 196 & & $6.2(4.8,7.6)$ & $7.0(4.5,9.5)$ & $0.8(-3.8,5.4)$ & 1.000 \\
\hline & Intervention III & 219 & & $7.5(5.8,9.3)$ & $10.9(9.5,12.3)$ & $3.4(0.9,5.9)$ & $<0.001$ \\
\hline & Intervention IV & 190 & & $11.2(9.3,13.1)$ & $9.6(7.5,11.6)$ & $-1.6(-4.3,1.0)$ & 1.000 \\
\hline \multirow[t]{7}{*}{ HEPTEM a $\left({ }^{\circ}\right)^{b}$} & Intervention I & 265 & \multirow[t]{4}{*}{90 days or less } & $77.0(73.7,79.7)$ & $36.6(31.2,42.2)$ & $-40.4(-50.4,-30.5)$ & $<0.001$ \\
\hline & Intervention || & 204 & & $42.1(37.8,46.4)$ & $74.9(72.1,77.4)$ & $32.9(23.2,42.5)$ & $<0.001$ \\
\hline & Intervention ||| & 217 & & $72.8(69.8,75.4)$ & $63.9(60.0,67.4)$ & $-8.9(-15.0,-2.8)$ & $<0.001$ \\
\hline & Intervention IV & 188 & & $64.4(57.6,70.3)$ & $70.2(64.0,75.2)$ & $5.7(0.3,11.2)$ & 0.038 \\
\hline & Intervention I & 265 & \multirow[t]{3}{*}{ Older than 90 days to 2 years } & $75.0(71.4,78.0)$ & $49.5(43.7,55.1)$ & $-25.5(-32.8,-18.3)$ & $<0.001$ \\
\hline & Intervention || & 204 & & $54.0(50.5,57.3)$ & $74.9(72.4,77.1)$ & $20.9(13.9,28.0)$ & $<0.001$ \\
\hline & Intervention III & 217 & & $72.4(69.7,74.8)$ & $67.9(64.7,70.8)$ & $-4.5(-8.2,-0.8)$ & $<0.001$ \\
\hline
\end{tabular}


Table 3 Estimated average changes in ROTEM values after four waves of interventions (blood product transfusions) (Continued)

\begin{tabular}{|c|c|c|c|c|c|c|c|}
\hline \multirow[t]{2}{*}{ Outcome } & \multirow[t]{2}{*}{ Intervention } & \multirow[t]{2}{*}{$N^{a}$} & \multirow[t]{2}{*}{ Age Group } & \multicolumn{3}{|c|}{ Estimated ROTEM Values } & \multirow[t]{2}{*}{$p^{b, c}$} \\
\hline & & & & Before $(95 \% \mathrm{Cl})$ & After (95\% Cl) & Change (95\% Cl) & \\
\hline & Intervention IV & 188 & & $67.8(61.1,73.3)$ & $68.7(61.6,74.5)$ & $0.9(-1.2,3.0)$ & 1.000 \\
\hline & Intervention I & 265 & Older than 2 years & $73.4(69.6,76.7)$ & $61.3(55.1,66.9)$ & $-12.1(-19.5,-4.8)$ & $<0.001$ \\
\hline & Intervention || & 204 & & $57.4(51.2,63.1)$ & $69.8(57.0,78.6)$ & $12.4(-3.3,28.1)$ & 1.000 \\
\hline & Intervention ||| & 217 & & $68.6(56.9,77.1)$ & $65.3(61.9,68.3)$ & $-3.4(-9.0,2.3)$ & 1.000 \\
\hline & Intervention IV & 188 & & $66.1(58.5,72.4)$ & $69.8(62.8,75.4)$ & $3.7(-1.0,8.4)$ & 1.000 \\
\hline
\end{tabular}

${ }^{\mathrm{a}} \mathrm{N}$ was number of observations used for each model. All the models were controlled for age groups, whether each of the interventions was received, as well as interaction between age groups and before or after the intervention

${ }^{\mathrm{b}}$ Generalized linear mixed model using beta distribution was applied to predict a $\left(^{\circ}\right)$, while generalized estimation equation was used for other outcomes. Estimates were predicted marginal means

${ }^{c} p$ values were Bonferroni-adjusted for estimated mean changes before and after the intervention in each age group

$\mathrm{Cl}$ confidence interval, $C T$ clotting time, MCF maximum clot firmness

$p$ values in bold represent statistical significance

indicates a deficiency in clotting factors that can be mitigated with the transfusion of FFP. A low HEPTEM $\alpha$ indicates platelet deficiency, and a low FIBTEM MCF indicates fibrinogen deficiency, so improvements in these parameters would be expected. Changes in ROTEM following platelet transfusion can be attributed to clotting factors and fibrinogen in the residual plasma in which the platelets are suspended; this rationale is supported by the observed average increase of $69.8 \mathrm{mg} / \mathrm{dL}$ in fibrinogen level.

The normal concentration of fibrinogen in plasma is $160-450 \mathrm{mg} / \mathrm{dL}$ [7-9]. HFC is indicated for the treatment of acute bleeding in patients with congenital fibrinogen deficiency [10]. The standard adult dose of HFC is $70 \mathrm{mg} / \mathrm{kg}$ when the fibrinogen level is known, but the dose of HFC can be calculated based on actual and target fibrinogen concentrations using the following formula [10]:

$$
\begin{aligned}
& \text { Dose }(\mathrm{mg} / \mathrm{kg} \text { body weight }) \\
& =\frac{\text { target level }(\mathrm{mg} / \mathrm{dL}) \text {-measured level }(\mathrm{mg} / \mathrm{dL})}{1.7(\mathrm{mg} / \mathrm{dL} \text { per } \mathrm{mg} / \mathrm{kg} \text { body weight })}
\end{aligned}
$$

In this study, treatment with HFC at a median dose of $70 \mathrm{mg} / \mathrm{kg}$ caused an average increase in the FIBTEM MCF of $2.3 \mathrm{~mm}$ and an average increase in fibrinogen concentration of $73.1 \mathrm{mg} / \mathrm{dL}$. A pilot study reporting use of a FIBTEM-guided protocol for administration of fibrinogen concentrate to target a high-normal plasma fibrinogen concentration in adult patients undergoing aortic valve operation and ascending aorta replacement demonstrated lower transfusion requirements and lower post-operative bleeding compared with patients receiving conventional transfusion management [11]. However, there is a lack of similar data in pediatric populations. Increased understanding of the typical or expected increases in FIBTEM MCF and fibrinogen concentration with HFC, such as those observed here, would assist clinicians in determining appropriate doses in pediatric patients.
A surprising observation in this study was the prolongation of the HEPTEM CT by $110.8 \mathrm{~s}$ and a decrease in the HEPTEM $\alpha$ by $5.6^{\circ}$ following HFC administration. Protamine administration is temporally related to HFC administration, and the reversal of heparin and the initiation of thrombus formation would, we suggest, utilize some of the in situ platelets and clotting factors.

All the observed impacts of interventions were magnified in the youngest patient group ( $\leq 90$ days of age). This would be expected since patients in this cohort would have immature coagulation system [12-14]. There are quantitative and qualitative differences in fibrinogen function between neonates and adults due to the presence of "fetal" fibrinogen [15]. Confocal microscopy has demonstrated that there are significant structural differences between adult and neonatal fibrin networks: neonatal fibrin lacks three-dimensional structure due to the absence of cross-linking, which occurs in adult fibrin networks, making the neonatal clot more porous and less stable [15]. Interestingly, even with treatment with adult fibrinogen, fibrin function is not fully restored [15].

It has previously been shown that use of ROTEM can reduce the need and amount of transfused blood products in pediatric cardiac surgery patients [16]. Additionally, Tirotta et al. have demonstrated that administering HFC at a dose of $70 \mathrm{mg} / \mathrm{kg}$ to neonates and infants undergoing cardiac surgery reduced FFP and cryoprecipitate requirements [17]. Targeting a high-normal FIBTEM MCF in this age group may lead to even further reductions in transfusion requirements. Further prospective trials are needed in the pediatric population to test this hypothesis.

\section{Conclusions}

This study demonstrates that CPB profoundly and negatively impacts all ROTEM values in pediatric patients undergoing cardiac surgery. Transfusion with platelets $(38 \mathrm{~mL} / \mathrm{kg})$ improved HEPTEM $\alpha$ by $22.1^{\circ}$ while FIBTEM MCF increased by $2.9 \mathrm{~mm}$. Administration of HFC 


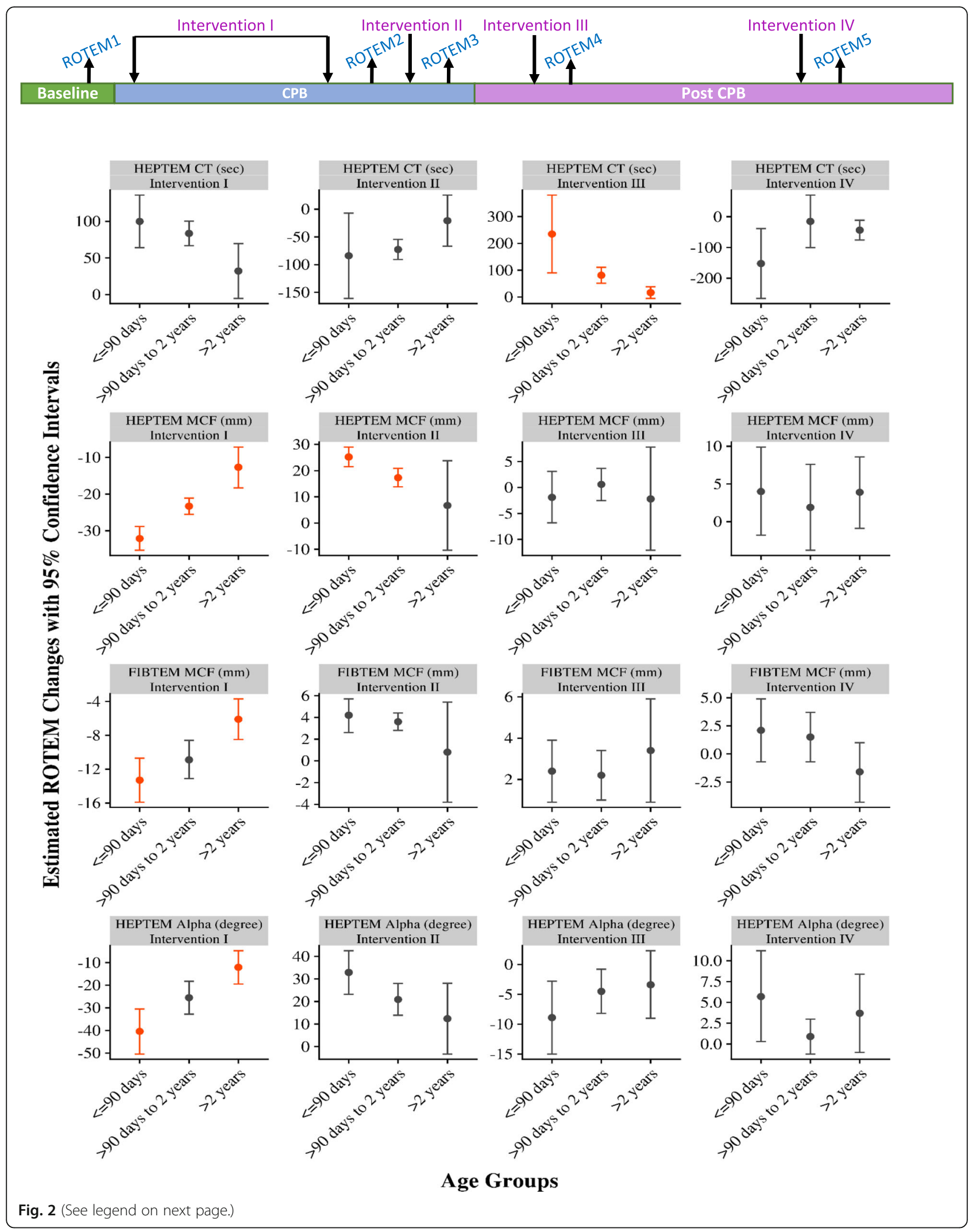


(See figure on previous page.)

Fig. 2 Estimated changes in ROTEM values by age groups. Estimated changes were predicted marginal means from generalized estimation equation models for HEPTEM MCF, HEPTEM CT, and FIBTEM MCF, and generalized linear mixed models for HEPTEM Alpha. A negative change indicates a decrease in the ROTEM value from before to after an intervention, whereas a positive change indicates an increase in the ROTEM value from before to after the intervention. The change is not significant if the $95 \%$ confidence interval crossed 0 . Pairwise comparisons of changes in ROTEM values were performed by age groups using 95\% confidence intervals. Age groups with significant difference were marked in red. $C$, clotting time; MCF, maximum clot firmness.

improved FIBTEM MCF by $2.7 \mathrm{~mm}$ and led to an average increase in fibrinogen concentration of $73 \mathrm{mg} /$ dL. Further research is necessary to corroborate these results.

\section{Abbreviations}

Cl: Confidence intervals; CPB: Cardiopulmonary bypass; CS: Cell saver; CT: Clotting time; EMC: Estimated mean change; FFP: Fresh frozen plasma; GEE: Generalized estimation equation; HFC: Human fibrinogen concentrate; IRB: Institutional Review Board; MCF: Maximum clot firmness;

PB: Phlebotomized blood; PLT: Platelets; POC: Point of care; PRBC: Packed red blood cells; ROTEM: Rotational thromboelastometry;

TEG: Thromboelastography

\section{Acknowledgments}

Editorial assistance was provided by Philip Chapman of Fishawack Communications Ltd., funded by CSL Behring.

\section{Authors' contributions}

CFT: Made primary contribution to study conception and design, data acquisition and analysis and drafting and revising the manuscript for intellectual content. RGL: Made substantial contribution to study conception and design, data acquisition and drafting and revising the manuscript for intellectual content. DS: Made substantial contributions to data analysis and drafting and revising the manuscript for intellectual content. WW: Made substantial contributions to data analysis and drafting and revising the manuscript for intellectual content. TT: Made substantial contributions to data analysis and drafting and revising the manuscript for intellectual content. JO: Made substantial contribution to data acquisition and drafting and revising the manuscript for intellectual content. KK: Made substantial contribution to data acquisition and drafting and revising the manuscript for intellectual content. HL: Made substantial contribution to data acquisition and drafting and revising the manuscript for intellectual content. $\mathrm{RH}$ : Made substantial contribution to drafting and revising the manuscript for intellectual content. RB: Made substantial contribution to drafting and revising the manuscript for intellectual content. All authors read and approved the final manuscript.

\section{Funding}

This manuscript received financial support from CSL Behring.

\section{Availability of data and materials}

The datasets used and/or analyzed during the current study are available from the corresponding author on reasonable request.

\section{Ethics approval and consent to participate}

This study received Institutional Review Board (IRB) exempt status from the Research Institute of Nicklaus Children's Hospital.

\section{Consent for publication}

Not applicable.

\section{Competing interests}

The primary author received financial support from CSL Behring for preparation of this manuscript. All other authors declare no competing interests.

\section{Author details}

'Department of Anesthesia, The Heart Program, Nicklaus Children's Hospital, 3100 S.W. 62nd Street, Miami, FL 33155, USA. ${ }^{2}$ Research Institute, Nicklaus Children's Hospital, Miami, FL, USA. ${ }^{3}$ Department of Cardiac Surgery, The
Heart Program, Nicklaus Children's Hospital, 3100 S.W. 62nd Street, Miami, FL 33155, USA.

Received: 18 December 2018 Accepted: 24 June 2019

Published online: 22 July 2019

\section{References}

1. Hartert H. Blutgerinnungsstudien mit der Thrombelastographie; einem neuen Untersuchungs verfahren. Klin Wochenschr. 1948;26:577-83.

2. Bolliger D, Seeberger MD, Tanaka KA. Principles and practice of thromboelastography in clinical coagulation management and transfusion practice. Transfus Med Rev. 2012;26:1-13.

3. Solomon C, Sørensen B, Hochleitner G, Kashuk J, Ranucci M, Schöchl H. Comparison of whole blood fibrin-based clot tests in thrombelastography and thromboelastometry. Anesth Analg. 2012;114:721-30.

4. Haas T, Spielmann N, Mauch J, Speer O, Schmugge M, Weiss M. Reproducibility of thrombelastometry $\left(\mathrm{ROTEM}^{\oplus}\right)$ : point-of-care versus hospital laboratory performance. Scand J Clin Lab Invest. 2012;72:313-7.

5. Ogawa S, Szlam F, Chen EP, Nishimura T, Kim H, Roback JD, et al. A comparative evaluation of rotation thromboelastometry and standard coagulation tests in hemodilution-induced coagulation changes after cardiac surgery. Transfusion. 2012;52:14-22.

6. Oswald E, Stalzer B, Heitz E, Weiss M, Schmugge M, Strasak A, et al. Thromboelastometry (ROTEM ${ }^{\oplus}$ ) in children: age-related reference ranges and correlations with standard coagulation tests. Br J Anaesth. 2010;105:827-35.

7. Asselta R, Tenchini ML, Duga S. Inherited defects of coagulation factor V: the hemorrhagic side. J Thromb Haemost. 2006:4:26-34.

8. Kreuz W, Meili E, Peter-Salonen K, Haertel S, Devay J, Krzensk U, et al. Efficacy and tolerability of a pasteurised human fibrinogen concentrate in patients with congenital fibrinogen deficiency. Transfus Apher Sci. 2005;32:247-53.

9. Standeven KF, Ariens RA, Grant PJ. The molecular physiology and pathology of fibrin structure/function. Blood Rev. 2005;19:275-88.

10. CSL Behring. RiaSTAP ${ }^{\oplus}$ prescribing information. 2016. http://labeling.cslbehring. com/PI/US/RiaSTAP/EN/RiaSTAP_Prescribing-Information.pdf. Accessed 4 June 2019.

11. Rahe-Meyer N, Pichlmaier M, Haverich A, Solomon C, Winterhalter M, Piepenbrock $\mathrm{S}$, et al. Bleeding management with fibrinogen concentrate targeting a high-normal plasma fibrinogen level: a pilot study. $\mathrm{Br} J$ Anaesth. 2009:102:785-92.

12. Andrew M, Paes B, Milner $R$, Johnston $M$, Mitchell $L$, Tollefsen DM, et al. Development of the human coagulation system in the full-term infant. Blood. 1987;70:165-72.

13. Bleyer WA, Hakami N, Shepard TH. The development of hemostasis in the human fetus and newborn infant. J Pediatrics. 1971;79:838-53.

14. Kuhle S, Male C, Mitchell L. Developmental hemostasis: pro- and anticoagulant systems during childhood. Sem Thromb Hemost. 2003;29:329-38.

15. Brown AC, Hannan RT, Timmins LH, Fernandez JD, Barker TH, Guzzetta NA. Fibrin network changes in neonates after cardiopulmonary bypass. Anesthesiology. 2016;124:1021-31.

16. Romlin BS, Wahlander $\mathrm{H}$, Berggren $\mathrm{H}$, Synnergren M, Baghaei F, Nilsson $\mathrm{K}$, et al. Intraoperative thromboelastometry is associated with reduced transfusion prevalence in pediatric cardiac surgery. Anesth Analg. 2011;112:30-6.

17. Tirotta CF, Lagueruela RG, Madril D, Ojito J, Balli C, Velis E, et al. Use of human fibrinogen concentrate in pediatric cardiac surgery patients. Int Anesthetic Anesthesiol. 2015:2:037.

\section{Publisher's Note}

Springer Nature remains neutral with regard to jurisdictional claims in published maps and institutional affiliations. 